\title{
Cauterización transoral de la arteria maxilar interna en la fosa infratemporal. Informe de un caso
}

\author{
Agustina Loreaø, Eduardo Figueroaø, Rocío Piastreliniø, Federico A. Urquiolaø, \\ Gabriel Rondón González ${ }^{\text {y }}$ Carlos Santiago Ruggeriø
}

\begin{abstract}
RESUMEN
Se describe el caso clínico de una paciente de 84 años que tuvo epistaxis recidivante por padecer enfermedad de Rendu-Osler-Weber. Tuvo antecedentes de diversos tratamientos quirúrgicos que incluyeron el cierre de la fosa nasal izquierda (operación de Young). Por la persistencia de epistaxis izquierda se indicó una angiografía y embolización. Esta última no se hizo porque se diagnosticaron anastomosis entre el sistema carotídeo externo y el interno.

Se realizó un abordaje intraoral paramaxilar asistido con endoscopios para cauterizar la arteria maxilar interna en la fosa infratemporal y un abordaje externo para cauterizar la arteria etmoidal anterior solucionando la epistaxis.
\end{abstract}

Palabras clave: arteria maxilar interna, abordaje transoral, endoscopios, fosa infratemporal, Rendu-Osler- Weber.

\section{TRANSORAL CAUTERIZATION OF THE INTERNAL MAXILLARY ARTERY IN THE INFRATEMPORAL FOSSA. CASE REPORT \\ ABSTRACT}

The clinical case of an 84-year-old patient who had recurrent epistaxis due to Rendu-Osler- Weber disease is described. She had a history of various surgical treatments including closure of the left nostril (Young's operation).

Due to the persistence of left epistaxis, angiography and embolization were indicated. The latter was not done because anastomosis between the external and internal carotid system was diagnosed.

An intraoral paramaxillary approach assisted with endoscopes was performed to cauterize the internal maxillary artery in the infratemporal fossa and an external approach to cauterize the anterior ethmoidal artery solving the epistaxis.

Key words: internal maxillary artery, transoral approach, endoscopes, infratemporal fossa, Rendu-Osler-Weber.

Rev. Hosp. Ital. B.Aires 2021; 41(3): 123-126.

\section{INTRODUCCIÓN}

El tratamiento quirúrgico de la epistaxis posterior se realiza habitualmente por vía endonasal con asistencia de endoscopios. Se diseca la arteria esfenopalatina, rama terminal de la arteria maxilar interna y se coagula con bipolar. Las arterias etmoidales también pueden ser cauterizadas bajo visión endoscópica mediante un abordaje transnasal localizando la arteria en la base del cráneo o en la órbita. La imposibilidad de acceder a estas arterias a través de las fosas nasales obliga a utilizar otros abordajes.

El acceso transoral subperióstico con endoscopios para cauterizar la arteria maxilar interna en la fosa infratemporal no es bien conocido y existen pocas referencias en la literatura.
Recibido: $18 / 6 / 21$

Aceptado: $26 / 8 / 21$

Servicio de Otorrinolaringología. Hospital Italiano de Buenos Aires. Departamento de Clínica Quirúrgica (C.S.R.), Instituto Universitario Hospital Italiano de Buenos Aires. Buenos Aires, Argentina

Correspondencia: carlos.ruggeri@hospitalitaliano.org.ar
Describimos el caso clínico de una paciente que tuvo epistaxis recidivante por padecer enfermedad de RenduOsler-Weber y que fue tratada con éxito mediante una cauterización de la arteria maxilar interna en la fosa infratemporal y de la arteria etmoidal anterior mediante un abordaje externo.

\section{CASO CLÍNICO}

Paciente de sexo femenino, de 84 años.

Tuvo antecedentes de epistaxis desde los 30 años y diagnóstico de telangiectasias hereditarias hemorrágicas o enfermedad de Rendu-Osler-Weber (criterios de Curaçâo 4/4).

Fue evaluada y en seguimiento por la Unidad de Telangiectasia Hemorrágica Hereditaria del Hospital Italiano de Buenos Aires durante los últimos 10 años.

Tuvo múltiples taponajes nasales, transfusiones, infusiones de hierro y de bevacizumab, y padeció telangiectasias y fístulas arteriovenosas hepáticas y pulmonares.

Fue tratada mediante embolización por malformaciones arteriovenosas pulmonares, y por su epistaxis (Sadick 2) mediante diferentes tratamientos quirúrgicos durante varios años. 
En agosto de 2013 se le realizaron infiltraciones de esclerosantes (polidocanol 0,5\%) en fosa nasal izquierda y dos años después, por la persistencia del sangrado, se hizo una septodermoplastia izquierda.

En el año 2018 se coagularon telangiectasias nasales izquierdas con radiofrecuencia (COBLATION).

En febrero del año 2020 tuvo una internación prolongada por epistaxis que se complicó con un paro cardiorrespiratorio de causa hipóxica secundario a la obstrucción de la vía aérea por coágulos.

Se le indicaron tapones de silicona para ocluir la fosa nasal izquierda.

En noviembre del año 2020 se realizó una oclusión de la fosa nasal izquierda con técnica de Young y un mes después, por la persistencia de epistaxis izquierda, se indicó una angiografía digital y embolización.

Se diagnosticó una hipervascularización en cavidades nasales izquierdas a expensas de la arteria esfenopalatina y de la arteria etmoidal anterior, rama de la arteria oftálmica. Se observaron shunts entre la circulación proveniente de la arteria carótida externa (arteria esfenopalatina) y de la carótida interna (arteria etmoidal anterior).

Por el riesgo de reflujo de las partículas que se utilizan para hacer la embolización desde el sistema carotídeo externo al interno (podrían provocar un accidente cerebrovascular) se prefirió realizar una cauterización de la arteria maxilar interna izquierda en la fosa infratemporal (II porción), por vía transoral con asistencia de endoscopios de $0^{\circ}$.

Se asoció en el mismo tiempo quirúrgico una cauterización de la arteria etmoidal anterior izquierda mediante un abordaje externo (Fig. 1).
La vía endonasal no pudo utilizarse por la oclusión previa de la fosa nasal izquierda.

La técnica quirúrgica de cauterización de la arteria maxilar interna por abordaje intraoral consistió en realizar, bajo anestesia general, una incisión con cauterio en forma de $\mathrm{J}$ a nivel del segundo molar superior, y disecar en un plano subperióstico hasta llegar a la curvatura posterior del seno maxilar.

A medida que se progresó en la disección se utilizaron separadores para mantener una adecuada visión en el corredor paramaxilar.

Se cauterizó durante el abordaje a la arteria alveolar. Se incidió el periostio y se disecó la grasa de la fosa infratemporal hasta observar el músculo pterigoideo externo.

En el extremo superior se expuso la arteria maxilar interna y se cauterizó con bipolar. Se cerró la herida con sutura reabsorbible (Fig. 2).

La arteria etmoidal anterior se cauterizó mediante un abordaje externo realizado por una incisión tipo Lynch en el ángulo interno del ojo, de aproximadamente $1,5 \mathrm{~cm}$. La paciente tuvo buena evolución con resolución de la epistaxis y sin trismo.

\section{DISCUSIÓN}

La arteria maxilar interna se origina de la carótida externa y atraviesa el ojal retrocondíleo de Juvara, entre el cuello de la mandíbula y el ligamento esfenomandibular (primera porción), y desde allí penetra en la región infratemporal, siguiendo un trayecto paralelo al músculo pterigoideo externo, que puede ser superficial o profundo (segunda porción).

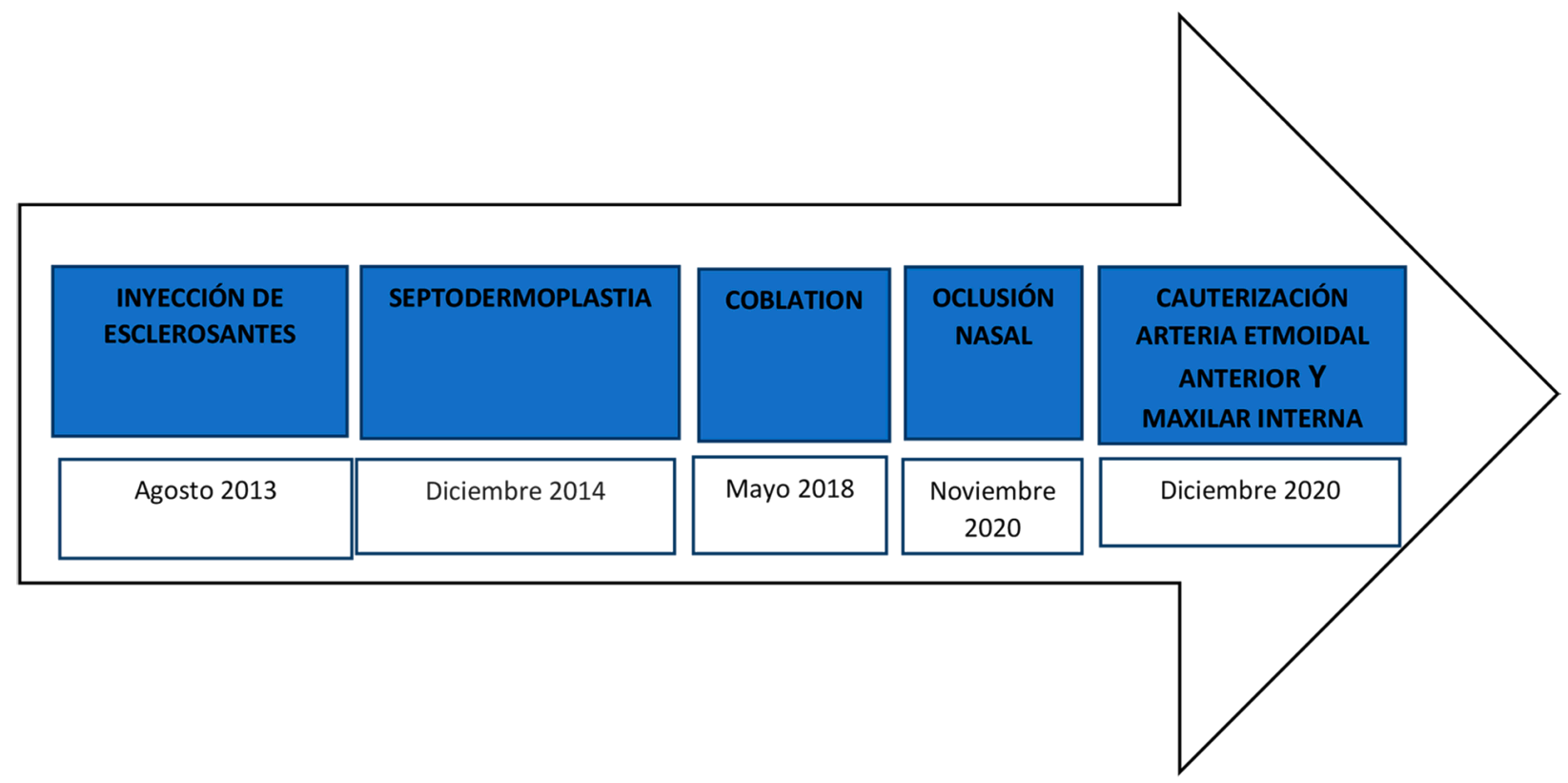

Figura 1. Tratamientos previos a la cauterización de la arteria maxilar interna y etmoidal anterior. 

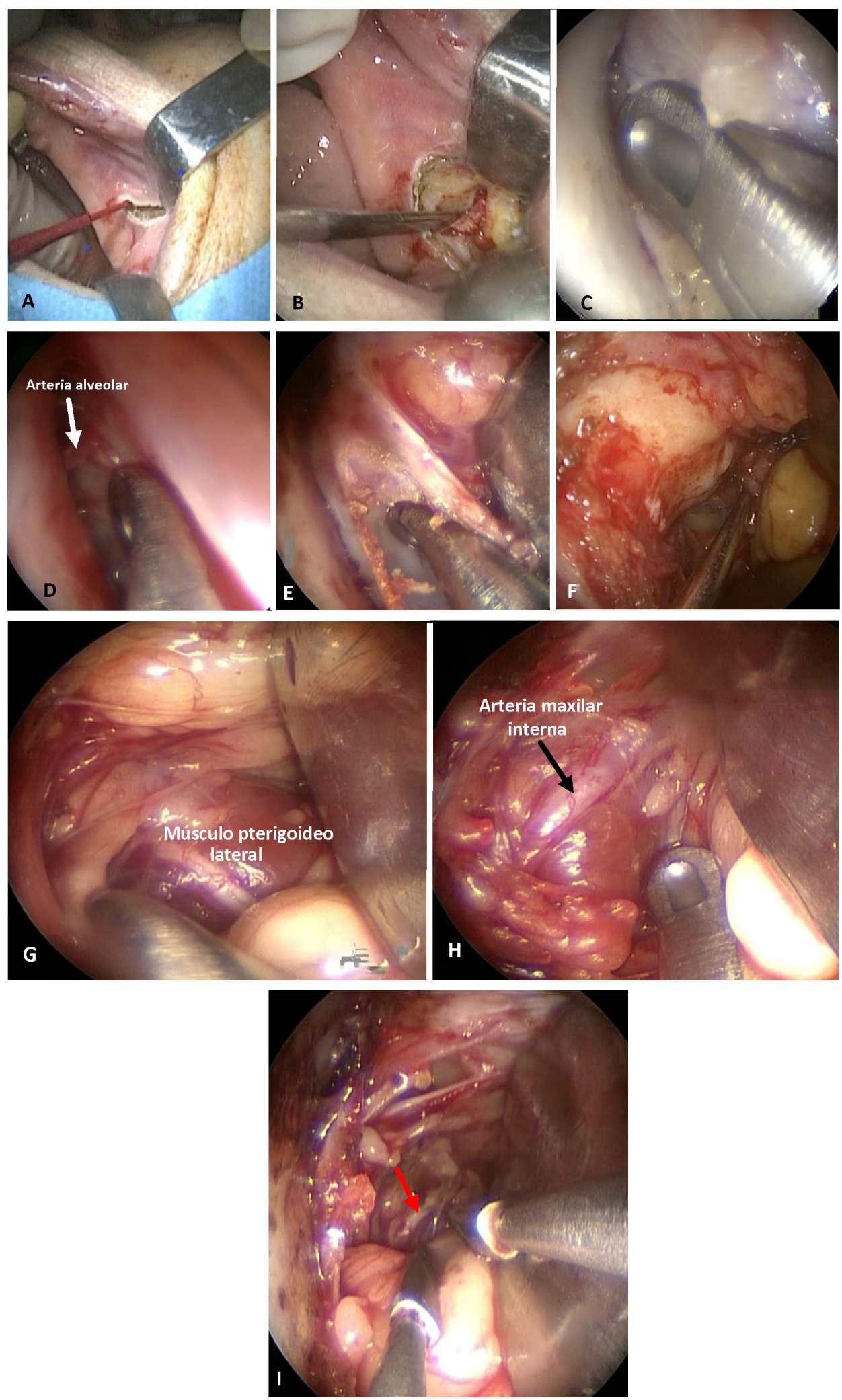

Figura 2. Cauterización transoral de la arteria maxilar interna con asistencia de endoscopios. Técnica quirúrgica: A) incisión en la mucosa gingivobucal superior, B-C) disección subperióstica paramaxilar, D) identificación y cauterización con bipolar de la arteria alveolar, E-F) apertura del periostio y disección de la grasa de la fosa infratemporal, G) identificación del músculo pterigoideo lateral, H) disección de la arteria maxilar interna (flecha) en la fosa infratemporal, I) cauterización con bipolar de la arteria maxilar interna (flecha). 
Ingresa después en la fosa pterigomaxilar y finaliza en la cavidad nasal como arteria esfenopalatina (tercera porción). Maceri y cols. fueron los primeros en describir la técnica de ligadura transoral de la arteria maxilar interna en 1984, en una serie de 13 pacientes. Ellos no aconsejaron la disección medial de la arteria maxilar interna alejada del músculo temporal porque esta -como variante anatómica- puede tener un trayecto posterior al músculo pterigoideo lateral. La disección más lateral puede asociarse a trismo severo que puede durar entre 2 y 3 meses ${ }^{1,2}$.

Sala y cols. ${ }^{3}$ modificaron esta técnica realizando una disección subperióstica asistida con endoscopios para localizar la arteria maxilar interna en la parte superior de la fisura pterigomaxilar, incluso en aquellos casos en que la arteria esté oculta por el vientre inferior del músculo pterigoideo lateral.

Para tratar la epistaxis o desvascularizar un tumor puede cauterizarse la arteria esfenopalatina mediante un abordaje endonasal $\mathrm{l}^{4,5} \mathrm{o}$, con menos frecuencia, se puede cauterizar la arteria maxilar interna accediendo a esta en la fosa pterigomaxilar mediante una amplia antrostomía maxilar media, resecando la pared posterior del seno maxilar6 ${ }^{6}$.
En el caso descripto, la oclusión de la fosa nasal izquierda por la cirugía previa imposibilitó el abordaje endonasal; además, la presencia de múltiples telangiectasias posiblemente hubiese dificultado el acceso por el sangrado de estas. Otras circunstancias que pueden favorecer el abordaje transoral son la presencia de tumores que ocupen la cavidad nasal o el seno maxilar y dificulten el acceso endonasal y transantral, fracturas faciales que originen epistaxis y malformaciones vasculares de la arteria maxilar interna. Una posible limitación de la técnica es la presencia de anastomosis entre la arteria maxilar interna y la carótida interna y las ramas arteriales contralaterales. Al cauterizar la arteria en su segunda porción es posible que el sangrado persista debido al flujo retrógrado o a la presencia de anastomosis ${ }^{7}$.

\section{CONCLUSIONES}

La disección subperióstica transoral y la coagulación de la arteria maxilar interna en su segunda porción con asistencia de endoscopios configuraron una técnica quirúrgica muy útil en la resolución de la epistaxis posterior ante el riesgo de la embolización y la imposibilidad de utilizar un abordaje endonasal.

Conflictos de interés: los autores declaran no tener conflictos de interés.

\section{REFERENCIAS}

1. Maceri MR, Makielski KH. Intraoral ligation of the maxillary artery for posterior epistaxis. Laryngoscope. 1984;94(6):737-41. doi: 10.1288/00005537-198406000-00002.

2. Stepnick DW, Maniglia AJ, Bold EL, et al. Intraoral-extramaxillary sinus approach for ligation of maxillary artery: an anatomic study with clinical correlates. Laryngoscope. 1990;100(11):1166-70. doi: 10.1288/00005537-199011000-00006.

3. Salas-Galicia JE, Garza-Talamas LM, López-
Vázquez R, et al. Abordaje paramaxilar transoral endoscópico a la fosa infratemporal y arteria maxilar. An Orl Mex. 2017; 62(3):182-96.

4. Abdelkader M, Leong SC, White PS. Endoscopic control of the sphenopalatine artery for epistaxis: long-term results. J Laryngol Otol. 2007;121(8):759-62. doi: 10.1017/S0022215106003379.

5. Snyderman CH, Goldman SA, Carrau RL, et al. Endoscopic sphenopalatine artery ligation is an effective method of treatment for posterior epistaxis. Am J Rhinol. 1999;13(2):137-140. doi: $10.2500 / 105065899782106805$.

6. Chandler JR, Serrins AJ. Transantral ligation of the internal maxillary artery for epistaxis. Laryngoscope. 1965;75:1151-59. doi: 10.1288/00005537-196507000-00011.

7. Polev GA, Carrau RL, Golbin DA, et al. Intraoral endoscopic ligation of maxillary artery in the infratemporal fossa. J Craniofac Surg. 2019;30(1):139-40. doi: 10.1097/ SCS.0000000000004981. 